\title{
A framework for assessing social- and location-based transmission risk as a heuristic for individual decision-making.
}

\author{
Carrie Diaz Eaton ${ }^{1, *}$
}

\section{Bates College}

* cdeaton@bates.edu

\begin{abstract}
This paper provides a framework for the assessment of household-level risk, incorporating both a individual social risk perspective and a location-based perspective. We use this framework as a heuristic to explore the effect of social reintegration choices individuals face, which are not be addressed by current policies. For example, we explore how integrating extended family households during COVID-19 without social distancing may affect household and community risk. The goal is to aid individual decision makers, who are seeking to maintain quality-of-life while navigating local policy, with nuance relating to location-specific behavior and disease prevalence.
\end{abstract}

\section{Introduction}

The COVID-19 pandemic has put the brakes on the world, both lives and economies. Now that countries are seeing new cases decline, re-opening economies safely has been on the agenda. How do we make decisions of how to act/where to go as we re-open? What are the ways we can assess our risk to others, others' risk to us, and the public health risk of joining household units?

As we make re-opening decisions, some individuals are informed by state-level policies and politics [1. But there is a large interpretation gap. In the wake of COVID-19, many papers analyzed individual risk of complications from COVID-19, e.g. [4]. They also analyzed risk for broader public policy e.g. [3]. So far, they have not been addressing individual concerns as states begin relaxing stay at home orders. While some current news articles have tried to provide some general advice, e.g. 5. 12, there is little in the way of assessing one's household-level risk of disease transmission when considering visits to friends and family.

The framework presented below is a simplified model which analyzes risk as a function of individual social behavior and location-based factors. This heuristic guide is not meant to override any policy level, and is limited by many simplifying assumptions used throughout. However, it may provide some intuition in how many levels of risk factors can behave together in an overall risk profile which can be used to compare risk across scenarios.

We use the assumption is that risk levels are multiplicative, i.e if risks are probabilities, these risks are independent. This assumption is used throughout the calculations below as we calculate a total risk, defined as the social risk * location-based risk. 
A spreadsheet which helps organize this information for easy calculation can be found in the following link Risk Tool. To use this Google Spreadsheet and make edits, make a copy of the spreadsheet.

The following sections focus on how to assess calculate the social/behavioral risk and the location-based risk. We use these to calculate a total risk which can be compared across households. We then calculate the relative risk of breaking social distancing to pool or merge with another household as social distancing measures.

\section{Individual Social Risk}

How do you social distance? What are your working conditions? How does that confer risk? We might assume some general categories of individual risk due to social behavior and then approximate a level of transmission risk from that risk-level.

\subsection{Individual social risk levels}

We consider 5 risk scenarios. One might also consider defining a continuous function, but for the purpose of easy individual use, individuals may find it easier to pick risk category rather than indicate a value. In the categories below, I attempt to connect a social distancing category with contact rates and transmission probabilities.

- Risk level 1: Quarantine with no contact except within the household. $(0 \%)$

- Risk level 2: Stay-at-home with essential contact only, remote workplace. (low exposure, low risk - $98 \%$ safety on contact, one contact/day $=2 \%$ )

- Risk level 3: Essential worker in an environment practicing strict social distancing measures or in shared housing practicing social distancing measures (high/consistent exposure, but of low risk - 98\% safety on contact, 20 contacts/day $=33 \%$ )

- Risk level 4: Practicing intermittent social distancing (low - medium exposure, but of high risk - $50 \%$ safety on contact, 2 contacts/day $=75 \%$ )

- Risk level 5: Essential worker in an environment practicing no social distancing measures or not practicing social distancing (consistent exposure, high risk - 50\% safety on contact, 20 contacts/day $=100 \%$ )

Note that nothing is ever $0 \%$ risk, but we round for simplification. One could, if desired, assume a $0.1 \%$ risk instead.

We use the variable $C_{h, i}$ to define the Social Risk level for household $h$, individual $i$. A household is defined as a unit in which there is no social distancing between individuals. Most people may assume it is all people living in a single house, but there are exceptions. For example, if someone is a caretaker for a family member in another household without distancing, this would be considered part of a single household instead of two households. If divorced parents sharing care-taking responsibility of children, we would consider both houses as a single household.

\subsection{Examples}

Below we consider a few example households. These example households will be used throughout the remainder of the paper.

Household 1: One adult is working from home remotely. Two children are at home, but schools are closed. A second adult is an essential employee in a business 
practicing social distancing and sanitation guidelines, and runs all essential errands. Household 1 members $1,2,3$ have risk level 1 , so $C_{1,1}=C_{1,2}=C_{1,3}=0$. Household 1 member 4 has risk level 3: $C_{1,4}=0.33$.

Household 2: Two family members in house A, who regularly care-take for child in house B. Because the care-taking is a regular interaction without social distancing, the members of house A and house B together comprise one household. As the child is not going out other than traveling between households, they are level 1. One adult in house $\mathrm{A}$ is level 1 and one is level 2 (leaves only for essential errands). The adult in house B is an essential worker at a an essential facility practicing social distancing, and so is level 3. Household members 1 and 2 have risk level 1 , so $C_{2,1}=C_{2,2}=0$. Household member 3 and 4 have risk levels $C_{2,3}=0.02$ and $C_{2,4}=0.33$, respectively.

Household 3: Two retired individuals, one of which assumes all essential errand risk. Household members, therefore, have social risk level 1 and $2, C_{3,1}=0$ and $C_{3,2}=0.02$, respectively.

Household 4: Three household members, but all work. One adult works at home, but still runs essential errands $\left(C_{4,1}=0.02\right)$. One adult works in a construction workplace that is low-risk and practices social distancing guidelines $\left(C_{4,2}=0.33\right)$. The third adult is an essential medical caretaker, but uses all social distancing guidelines $\left(C_{4,3}=0.75\right)$.

\section{Household-level Social Risk}

The household-level social risk must amalgamate all of the above practices of individuals within a household. We assume that there is no quarantining going on within a house, but rather the household population is interacting consistently in ways conducive to transmitting any infection.

\subsection{Maximum risk conferred}

The simplest method is to assume that the risk level of a household is the maximum risk level across all individuals within the household.

$$
C_{h}=\max (C h, i \mid i \in \text { household } h)
$$

Using Eq. 1 for our example households we get the following household-level social risk assessments:

$$
\begin{aligned}
& C_{1}=\max (\{0,0,0,0.33\})=0.33 \\
& C_{2}=\max (\{0,0,0.02,0.33\})=0.33 \\
& C_{3}=\max (\{0,0.02\})=0.02 \\
& C_{4}=\max (\{0.02,0.33,0.75\})=0.75 .
\end{aligned}
$$

The advantage to this method is that it is relatively quick to assess as long as you know the risk level of the household member with the highest social risk profile. The disadvantage is that it can underestimate risk is households with multiple members with high social risk levels.

\subsection{Multiplicative risk}

Another variation is calculate the risk as a probability of contamination given that risk is like a probability of having a disease. To calculate, one would assume that the 
probability each individual is not contagious is $1-C_{h, i}$ and define risk as 1 - the probability that no individuals within the household are infected.

$$
C_{h}=1-\Pi\left(1-C_{h, i} \mid i \in \text { household } h\right)
$$

Using Eq. 2 for our example households we get the following household-level social risk assessments:

$$
\begin{aligned}
& C_{1}=1-(1-0)^{3}(1-0.33)=0.33 \\
& C_{2}=1-(1-0)^{2}(1-0.02)(1-0.33)=0.3434 \\
& C_{3}=1-(1-0)(1-0.02)=0.02 \\
& C_{4}=1-(1-0.02)(1-0.33)(1-0.75)=0.84 .
\end{aligned}
$$

Comparing two risk methods, there is little difference for households with most members at Level 1 or 2. Making the simplifying assumption about a maximum risk conferred has more effect on the risk of household with few at home members.

\section{Location-based Risk}

Two households such as Household 1 and Household 2, even with approximately the same social risk levels may be different in total risk due to a number of location-based factors. This includes disease prevalence as well as location-based social distancing behaviors, which could be a function of policy, but not necessarily. For example: About what percent of the population is using social distancing? What are the state or county guidelines for the level of openness? How well are the other residents in that county adhering to guidelines?

We define total location-based risk $=$ Location-based risk level $*$ Prevalence of disease at location. Below we introduce location-based risk levels and how to capture prevalence data consistently for the purposes of this relative risk assessment.

\subsection{Location-based Risk Levels}

Again, the simplest way to quantify location-based risk might be through some sort of risk level analogous to the social-risk levels. For simplification, we present risk levels the same as considered above, with the same way of conceptualizing the numerical risk score.

- Risk level 1: Strict stay at home, enough testing, contact tracing, quarantine of all contacts and suspected cases. (0\%)

- Risk level 2: Stay-at-home with essential contact only, remote workplace, no schools in session, all essential businesses with strict social distancing and disinfecting measures. (low exposure, low risk - $98 \%$ safety on contact, one contact $/$ day $=2 \%$ )

- Risk level 3: Business open but all practicing social distancing or predominately stay at home with a non-negligible percent of the population not social distancing (high/consistent exposure, but of low risk - 98\% safety on contact, 20 contacts/day $=33 \%$ )

- Risk level 4: Only partial practicing of social distancing (low - medium exposure, but of high risk - 50\% safety on contact, 2 contacts $/$ day $=75 \%$ ) 
- Risk level 5: Business as usual - Not practicing social distancing and no testing/quarantining (consistent exposure, high risk - 50\% safety on contact, 20 contacts/day $=100 \%)$

Again, note that nothing is ever $0 \%$ risk, but we round for simplification. One could, if desired, assume a $0.1 \%$ risk instead.

We define the variable $K_{j}$ as the location-based risk level in location $j$. A location could be a zip code, a city/town, a county, or a state. For all calculations that follow for a particular location, be sure to make scope of that location consistent.

\subsection{Examples}

Below we consider a few example locations. These example locations will be used throughout the remainder of the paper. The estimates of risk level and the conditions described throughout the paper were current as of May 15th, 2020.

Location 1: Cumberland County, Maine. Maine records disease data at the county level and makes policy both at the town, county, and state level. The state is on a stay-at-home order, with minimal exceptions for essential work at locations employing recommended social distancing and sanitation practices. Essential food shopping, health care, and limited outdoor quality-of-life activities are also permitted. Some rural counties were allowed to ease more significantly than some higher impacted counties.

Cumberland County is considered a county with community transmission and as such, no major restrictions were lifted. Cumberland County might be considered a risk level of $2, K_{1}=0.02$.

Location 2: Hancock County, Maine. Hancock County is one of the rural counties in Maine that has lifted additional restrictions due to low case prevalence.

Socially-distant shopping and in-restaurant dining are now permitted. Due to the low case numbers and density, some residents are not employing measures such as mask-wearing and social distancing. We categorize this as a risk level $4, K_{1}=0.75$.

Location 3: Warwick, Rhode Island, zip code 02889. Rhode Island data has been shared on their Health Department website at both the city/town and zip code levels. Rhode Island has had a higher case incidence overall than Maine, and many restrictions are in place, but not all residents in 02889 are following guidelines, sometimes congregating in groups without masks. We categorize this as a risk level $3, K_{1}=0.33$.

\subsection{Location-based Prevalence}

Disease prevalance is the number of cases of infected individuals at a given time. In order to be able to compare prevalance across many locations, we will use the number of active positive cases per capita, or $I_{j} / N_{j}$ where $I_{j}$ is the number of infecteds and $N_{j}$ is the number of residents in location $j$.

Unfortunately, cases refers to positive testing, which is not necessarily the same as the number of infected individuals. A recent serology study out of Spain indicates that one out of every 10 individuals with COVID-19, was identified through testing [6]. Therefore, we define disease prevalence, $P$ in location $j$ as the number of active cases reported per capita $* 10$, or

$$
P_{j}=10 * I_{j} / N_{j}
$$

Using Eq. 3, current active case data from the Maine website 7 and 2019 population data [8], we get the following per capita prevalence estimates for location 1 and 2 in Maine:

$$
\begin{aligned}
& P_{1}=823 / 295003 * 10=0.0279 \\
& P_{2}=10 / 54811 * 10=0.00182 .
\end{aligned}
$$


The Rhode Island data already provides per capita case estimates, in the form of the number of confirmed positive cases per 100,000 individuals [11]. The issue with this data for comparison with Maine is that we do not know how many cases are active versus recovered or deceased. While imperfect, we make the assumption that half the cases are active. In this case, we get a per capita prevalance estimate for location 3 :

$$
P_{3}=607 / 100000 * 0.5 * 10=0.0304
$$

\subsection{Total Location-Based Risk}

Recall that we defined total location-based risk in location $j$ as

$$
L_{j}=K_{j} * P_{j}
$$

Using Eq. 4 in our example locations, we get the following total location-based risk estimates:

$$
\begin{aligned}
& L_{1}=K_{1} * P_{1}=0.02 * 0.0279=0.000558 \\
& L_{2}=K_{2} * P_{2}=0.75 * 0.00182=0.001365 \\
& L_{3}=K_{3} * P_{3}=0.33 * 0.0304=0.001
\end{aligned}
$$

\section{Assessing the Risk of Pooling Households}

As we begin to relax our social behaviors, particularly where they relate to quality-of-life, we may be considering scenarios which merge households, either short-term or long-term. If these households are in the same location, we could compare just household-level risk. However, in cases where two households might span counties or even state-lines, such as traveling to see grandparents and give hugs, traveling to a new location brings local-level risk, mediated by their socially-distancing practices.

\subsection{Location-based Household-level Risk}

We now define the total location-based household-level risk as household-level social risk * total location-based risk or

$$
T_{h, j}=C_{h} * L_{j}
$$

Assume that Household 1 in is Location 1, Households 2 and 3 are in Location 2, and Household 4 is in Location 3. Using Eq. 5 for our example households we get the following household-level social risk assessments:

$$
\begin{aligned}
& T_{1,1}=C_{1} * L_{1}=0.33 * 0.000558=0.000184 \\
& T_{2,2}=C_{2} * L_{2}=0.3434 * 0.001365=0.00047 \\
& T_{3,2}=C_{3} * L_{2}=0.02 * 0.001365=0.0000273 \\
& T_{4,3}=C_{4} * L_{3}=0.84 * 0.001=0.00084 .
\end{aligned}
$$

\subsection{Pooled Risk}

Now suppose you want to travel to visit extended family or want to invite people over for a birthday party, without social distancing. When you do this, you assume the risk of their household. We will call this pooling households. If you combine or pool households, even temporarily, what is the effect?

As in the above pooling of individuals into a household, likewise, we treat the households as individuals in a larger pool. This assumes that each household has some 
risk probability, and we are calculating the complement of the probability that none of them bring infection.

$$
T_{p} \text { ool }=1-\Pi\left(1-T_{h, j} \mid h \text { households } \in \text { pool }\right)
$$

If we pool Households $1,2,3$, and 4 , the pooled risk is

$$
T_{\text {pool }}=1-(1-0.000184)(1-0.00047)(1-0.0000273)(1-0.00084)=0.00152 .
$$

\subsection{Risk Multiplier}

Keep in mind that the risk levels calculated so far are mostly useful for comparing relative risks across households and locations. When we assess how our risk changes based on an action scenario such as the pooling of households, we might calculate that change as how many times your risk increases in that scenario. In the case of pooling households, the risk multiplier for a particular household would be the pooled risk /total location-based household risk, or

$$
M_{h}=T_{p} \text { ool } / T_{h, j}
$$

Using Eq. 7, the risk multiplier in our household models would be:

$$
\begin{aligned}
& M_{1}=0.00152 / 0.000184=8.26 \text { times } \\
& M_{2}=0.00152 / 0.00047=3.23 \text { times } \\
& M_{3}=0.00152 / 0.0000273=55.68 \text { times } \\
& M_{4}=0.00152 / 0.00084=1.81 \text { times. }
\end{aligned}
$$

\subsection{Strategies for Reducing Risk}

Given the above scenario, it seems unlikely that Households 1-4 should all get together for a non-social distanced birthday party right now, particularly if Household 3 has any individuals at risk for complication.

One way to decrease risk would be to come together in a socially distanced way mediating the effect of pooling households. Another option would be to have high risk individuals and/or households quarantine as much as possible before coming together. Both would be required for a household coming from Location 3, Rhode Island, if such a gathering were to occur in the state of Maine. Under current policy out-of-staters must quarantine for 14 days, but this would not apply to Households 1 or 3 and so these decisions would be a voluntary action. However, both of these actions would significantly decrease risk.

Another option might be to have smaller gatherings. Again, this is consistent with the State of Maine recommendations which ask that gatherings be limited to 10 or under. What if we reduce the number of households that congregate?

Let us now consider if we limit the gatherings to like-risk households. If just the two lowest risk households gather, Households 1 and 3, the new pooled risk is $T_{\text {pool }}=0.000211$. This would change the risk mutliplier in those households to $M_{1}=1.15$ and $M_{3}=7.73$. If limited gatherings to the two highest risk households, Households 2 and 4 , the new pooled risk is $T_{\text {pool }}=0.00131$. This would change the risk mutliplier in those households to $M_{2}=2.79$ and $M_{4}=1.56$.

This solution would mean an overall smaller risk for all versus the full pooled risk and comply with current state-level policy. In particular, this solution significantly lowers the risk multiplier of Household 3 from around 56 times the risk to only 8 . 


\section{Risk to Public Health}

We assume that the risk to public health is that each household returns back to the original location with the increased transmission risk now assumed by any temporary pooling. This would increase the community transmission would be mediated by the social risk level. For example, bringing back a higher chance of infection if you are in strict quarantine or if you live in a location with strict location-based policies, may have little impact on overall public health. However, bringing a higher chance of infection to a location into a community with relaxed location-based policies or by an households with high social risk may impact community transmission levels 2 .

Meeting in a location with low community transmission might seem optimal to reduce any increased risk that pooling households might bring. However, the social distancing behavior of the location meeting should be taken into consideration. One must also consider the limited resources a community may have to deal with an outbreak. Bringing high-risk into a community that operates with low social distancing (due to lack of disease, cultural practices, or policy) that also has a low capacity for dealing with cases (for example as a result of structural racism and/or in rural communities) could present a serious public health concern 9.10. Ideally, households that make decisions to pool should consider absorbing the temporary higher risk by quarantining during and after such a gathering to prevent community transmission in high-risk locations.

\section{Acknowledgments}

We thank peer reviewers reading arxiv for their suggestions. We hope this framework will spur more efforts to help individuals assess their risks during COVID-19.

\section{References}

1. J. M. Barrios and Y. Hochberg. Risk perception through the lens of politics in the time of the covid-19 pandemic. Technical report, National Bureau of Economic Research, 2020.

2. S. Barua et al. Understanding coronanomics: The economic implications of the coronavirus (covid-19) pandemic. SSRN Electronic Journal https://doi org/10/ggq92n, 2020.

3. R. S. D'Amico, G. Baum, Y. Serulle, D. Silva, M. L. Smith, R. A. Wallack, J. A. Ellis, M. Levine, R. Ortiz, J. A. Boockvar, et al. A roadmap to reopening a neurosurgical practice in the age of covid-19. World Neurosurgery, 2020.

4. A. Gasmi, S. Noor, T. Tippairote, M. Dadar, A. Menzel, and G. Bjørklund. Individual risk management strategy and potential therapeutic options for the covid-19 pandemic. Clinical Immunology, page 108409, 2020.

5. G. Graham. When can i reunite with family members and friends? Portland Press, 2019.

6. Instituto de Salud Carlos III. Estudio ene-covid19: Primera ronda. estudio nacional de sero-empidemiología de la infeccíon por sars-cov-2 en españa, 2019. https://www.lamoncloa.gob.es/serviciosdeprensa/notasprensa/ sanidad14/Documents/2020/130520-ENE-COVID_Informe1.pdf, Last accessed on 2019-5-19. 
7. Maine Center for Disease Control and Prevention, Division for Disease Surveillance. 2019 novel coronavirus - (covid-19) - updates and information, 2019. https://www.maine.gov/dhhs/mecdc/infectious-disease/epi/airborne/ coronavirus.shtml, Last accessed on 2019-5-18.

8. Maine Center for Workforce Research and Information. County profiles, 2019. https://www.maine.gov/labor/cwri/county-economic-profiles/ countyProfiles.html, Last accessed on 2019-5-18.

9. E. M. Myers. Compounding health risks and increased vulnerability to sars-cov-2 for racial and ethnic minorities and low socioeconomic status individuals in the united states. 2020.

10. J. Solis, C. Franco-Paredes, A. F. Henao-Martínez, M. Krsak, and S. M. Zimmer. Structural vulnerability in the united states revealed in three waves of novel coronavirus disease (covid-19). The American Journal of Tropical Medicine and Hygiene, page tpmd200391, 2020.

11. State of Rhode Island Department of Health. Coronavirus disease 2019 (covid-19), 2019. https://health.ri.gov/diseases/ncov2019/, Last accessed on 2019-5-18.

12. L. Times. So you really want to see your friends - here's how to assess the risk. L.A. Times, 2019. 